\title{
Singlet-Triplet Transition in lateral Quantum Dots: A Numerical Renormalization Group Study
}

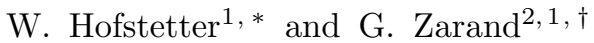 \\ ${ }^{1}$ Lyman Laboratory, Harvard University, Cambridge, MA 02138, USA \\ ${ }^{2}$ Theoretical Physics Department, Budapest University of Technology and Economics, Budafoki út 8. H-1521 Hungary
}

\begin{abstract}
We discuss transport through a lateral quantum dot in the vicinity of a singlet-triplet spin transition in its ground state. Extracting the scattering phase shifts from the numerical renormalization group spectra, we determine the linear conductance at zero temperature as a function of a Zeeman field and the splitting of the singlet and triplet states. We find reduced low-energy transport, and a non-monotonic magnetic field dependence both in the singlet and the triplet regime. For a generic set of dot parameters and no Zeeman splitting, the singlet-triplet transition may be identified with the conductance maximum. The conductance is least sensitive to the magnetic field in the region of the transition, where it decreases upon application of a magnetic field. Our results are in good agreement with recent experimental data.
\end{abstract}

\section{INTRODUCTION}

Recent observations of the Kondo effect in semiconductor quantum dots 1.2 induced enormous theoretical and experimental activity. Compared to magnetic impurities in solids $\frac{3}{3}$, quantum dots ${ }^{4}$ have the great advantage of tunable couplings, and as a result, in these systems the full parameter regime of the single-level Anderson impurity model can be systematically explored. In addition, new types of Kondo systems have been realized, like Aharonov-Bohm rings containing quantum $\operatorname{dots}^{5}$ and multi-level dots ${ }^{6}$. In vertical dots, a qualitatively new type of Kondo effect associated with a singlettriplet degeneracy has been observed ${ }^{7}$, which was later also explained theoretically ${ }^{8,9,10}$.

For lateral dots, this singlet-triplet Kondo effect has been found as well11 , but with qualitatively new behavior at low energies, where both the linear conductance and $d I / d V$ were found to be non-monotonic. Theoretical understanding of this behavior has been obtained in two limiting cases: Hofstetter and Schoeller 12 have analyzed the interplay between singlet and triplet configurations with symmetric coupling to the leads, while Pustilnik and Glazman ${ }^{13}$ have considered general asymmetric couplings but took only the triplet configuration on the dot into account.

Agreement with the singlet-triplet scenario of [12] has recently been found in another experiment $\frac{14}{4}$, where - instead of the magnetic field as in 11] - the Stark effect due to the gate potential was used to tune the level splitting. Another way to control the level spacing and to study the triplet-singlet transition by a symmetric (triangular) arrangement has been proposed in [15].

Our goal in this work is to extend the studies of 1213 over the entire range of parameters in a non-perturbative way, and to include singlet/triplet degeneracy as well as asymmetry of the tunnel couplings in our transport calculation. To this purpose we shall use the numerical renormalization group (NRG) in a rather unconventional way: We shall extract the $T=0$ scattering phase shifts directly from the NRG spectrum ${ }^{16.17}$ and use these to compute the conductance by applying the Landauer-Büttiker formula. Our calculations are in good agreement with experiments and reproduce the results in special limits as obtained in [12] and [13].

\section{THE MODEL}

In this paper we focus our attention to the lateral quantum dot system shown in Fig. 1. Such a quantum dot is usually formed by gate depletion in a two-dimensional electron structure. The conductances between the dot and the leads can be controlled by applying a voltage on the gates separating the dot region from the leads. In this system Coulomb blockade develops when the dimensionless conductances $g_{L, R} \equiv G_{L, R} /\left(2 e^{2} / h\right)$ between the dot and the left and right leads drop below 1: In this regime, electrons have to pay typically an energy of the order of the charging energy $\sim E_{C}$ to get onto the dot, and therefore transport through the structure is suppressed when the temperature drops below $E_{C}$. Since Coulomb blockade develops when the lowest propagating mode in the point contacts (between dot and leads) is at pinchoff, all higher modes can be neglected. The leads can thus be modeled by a single conduction electron mode each $^{18}$. Note that this is specific to lateral dot systems: In vertical dots (see e.g. 7] 9 ), many conducting modes participate in transport.

The Kondo effect may appear at temperatures $T \ll$ $E_{C}$ if the ground state of the isolated dot is degenerate. In this case quantum fluctuations to the leads are relevant, and may lead to the formation of a Kondo state at an energy scale $T_{K}$. This condition is obviously fulfilled if there is an odd number of electrons on the dot, since then the ground state of the dot has a Kramers degeneracy. The Kondo effect then emerges if we lower the temperature below the Kondo temperature 19

$$
T_{K} \sim \delta E \exp \left\{-E_{C} /\left(g_{L}+g_{R}\right) \delta E\right\}
$$

where $\delta E$ is the mean single-particle level spacing in the dot. 


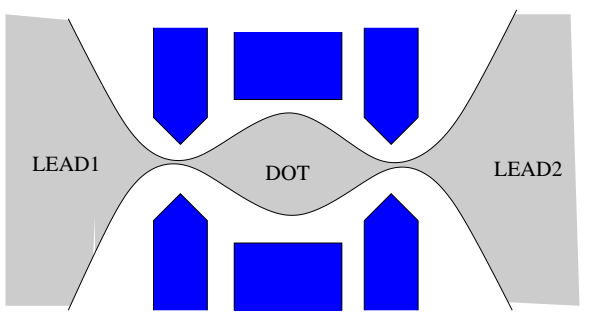

FIG. 1: Lateral quantum dot geometry. The dot is formed by depletion of the two-dimensional electron gas underneath the gates. Dark areas denote the positions of the gate electrodes, while the black lines indicate the border between depleted (white) and undepleted (grey) regions of the 2DEG.

However, Kondo effect can also be observed in dots with an even number of electrons. To understand the physics that leads to the Kondo effect in this case, let us first study an isolated quantum dot. A generic model of an isolated quantum dot can be written $\operatorname{as}^{20}$

$$
H_{\mathrm{dot}}=\sum_{n s} \epsilon_{n} d_{n s}^{\dagger} d_{n s}+E_{C}\left(\hat{N}-N_{0}\right)^{2}-E_{S} \hat{\mathbf{S}}^{2}-B \hat{S}^{z},
$$

where $\hat{N}=\sum_{n s} d_{n s}^{\dagger} d_{n s}$ is the total number of electrons on the dot, and $\hat{\mathbf{S}}=\sum_{n s s^{\prime}} d_{n s}^{\dagger}\left(\hat{\boldsymbol{\sigma}}_{s s^{\prime}} / 2\right) d_{n s^{\prime}}$ is the total electronic spin on the dot. The operators $d_{n s}^{\dagger}$ create electrons on a single particle level of the dot, labelled by the spin $s$ and a discrete quantum number $n$. Eq. (2) describes the electron-electron interaction at the mean field level. In general, more complicated interaction terms should be present in $H_{\text {dot }}{ }^{21}$. These terms are, however, relatively small for dots with a large number of electrons and furthermore, they do not influence our discussion of the singlet triplet transition below, therefore we shall neglect them.

The parameter $N_{0}$ in Eq. (2) denotes the dimensionless gate voltage, and it sets the average number $\langle\hat{N}\rangle$ of electrons on the dot, $B$ is the Zeeman field, and $E_{C}$ and $E_{S}$ stand for the charging energy and the Hund's rule coupling, respectively. In the rest of the paper we shall focus our attention to the case where $N_{0}$ is close to an even integer, and we shall assume $E_{C} \gg \delta E>E_{S}$, characteristic of lateral dots with a large number of electrons ${ }^{20}$. Under these conditions, the ground state of Eq. (2) is typically a singlet state shown on the left in Fig. 2] and therefore no Kondo effect occurs. If, however, the last occupied $\left(\epsilon_{-1}\right)$ and the first empty state $\left(\epsilon_{+1}\right)$ happen to be close enough to each other, so that $\Delta \equiv \epsilon_{+1}-\epsilon_{-1}<2 E_{S}$, then the system will form a triplet state to gain energy from the Hund's rule coupling by rearranging the level occupancy (see Fig. 2). In this case the ground state is threefold degenerate and a Kondo state can be formed. This transition occurs when the energy difference between the singlet and triplet states approximately vanishes:

$$
\Delta_{\mathrm{ST}} \equiv \epsilon_{+1}-\epsilon_{-1}-2 E_{S} \approx 0 .
$$

The Kondo effect is driven by virtual charge fluctua-
Singlet state $(\mathrm{S}=0)$

$\Delta$
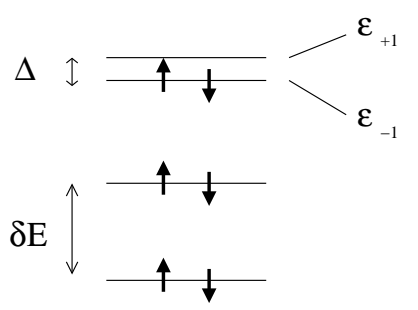

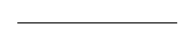

Triplet state $(\mathrm{S}=1)$

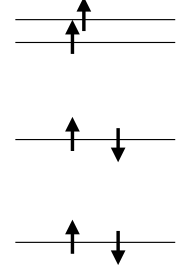

FIG. 2: Possible triplet and singlet ground states of an isolated dot described by Eq. (2).

tions to the leads. Since the coupling between the leads and the dot is weak, $g_{L}, g_{R} \ll 1$, we shall describe these fluctuations in terms of the following simple tunneling Hamiltonian:

$$
\begin{aligned}
H_{\text {leads }} & =\sum_{\alpha k s} \xi_{k} a_{\alpha k s}^{\dagger} a_{\alpha k s}, \quad \alpha=R, L \\
H_{\text {tunneling }} & =\sum_{\alpha n k s} t_{\alpha n} a_{\alpha k s}^{\dagger} d_{n s}+\text { H.c. }
\end{aligned}
$$

The operators $a_{\alpha k s}^{\dagger}$ in Eq. (3) create electrons with momentum $k$, energy $\xi_{k}$ and spin $s$ in the left and right leads $(\alpha=L, R)$, respectively. Eq. (44) describes the tunneling between the dot states (labelled by the spin $s$ and a discrete quantum number $n$ ) and the leads.

In the vicinity of the singlet-triplet transition charge (and spin) fluctuations on the dot are dominated by fluctuations to the states $n= \pm 1$. It is therefore sufficient to restrict the summations in Eqs. (2) and (4) and keep only these two states:

$$
\epsilon_{n}=n \Delta / 2, \quad n= \pm 1
$$

This projection will not crucially influence our results, and the two-level model is expected to capture all universal aspects of the problem until the relevant energy scales of the system that govern the singlet-triplet transition (i.e., the Kondo scales, $B$, and $\Delta_{\mathrm{ST}}$ ) are all small compared to all other energy scales $\left(E_{C}, \delta E\right.$ etc.) in the problem. However, when projecting to these two states, one also has to keep in mind that at energy scales larger than $\max \left\{\delta E, E_{C}\right\}$ fluctuations the the excited states of the dot destroy those coherent contributions that give rise the Kondo effect. Therefore, at the same time the effective band-width in Eq. (4) has to be reduced to $D \equiv \max \left\{\delta E, E_{C}\right\}^{22}$.

In the following sections we shall study the conductance of the dot when one gradually drives the system through the triplet singlet transition. There are several experimental techniques to achieve this goal and tune $\Delta$ 
and thus $\Delta_{\mathrm{ST}}$. The easiest method is to apply a magnetic field $H_{\perp}$ perpendicular to the plane of the dot. The primary effect of $H_{\perp}$ is to change the orbital wave functions and thus to control the value of $\Delta\left(H_{\perp}\right) \stackrel{7.11}{\underline{1}}$ By linearizing $\Delta\left(H_{\perp}\right)$ in the vicinity of the transition, one can thus attempt to make a direct comparison of the experimental data with the (calculated) linear conductance across the system $G=\left(2 e^{2} / h\right) g(\Delta)$. Unfortunately, $H_{\perp}$ also induces a Zeeman field $B\left(H_{\perp}\right)$ : While this Zeeman splitting $B$ remains small compared to the orbital effect because of the smallness of the electron effective mass and the $g$-factor in $\mathrm{GaAs}^{23}$, yet it is comparable to the experimentally observed Kondo scale on the triplet side ${ }^{11}$, and leads to some complications when trying to make a direct comparison with the experimental data. The difficulties related to Zeeman splitting were avoided in the experiment [14] by tuning the singlet-triplet transition via the Stark effect due to an inhomogeneous gate potential.

Another way to avoid this problem is to use triangular dots where the degeneracy of the states $\epsilon_{ \pm 1}$ can be directly controlled by changing the shape of the $\operatorname{dot}^{15}$. To investigate the dependence of $g(\Delta, B)$ on $B$ experimentally, one may in addition apply a strong in-plane field $H_{\|}$, which only generates a Zeeman splitting without influencing $\Delta$ essentially.

With the method outlined in Section IV] we cannot compute the finite temperature conductance of the dot. However, the temperature $(T)$, source-drain bias $(V)$, and Zeeman field $(B)$ dependences of the conductance are expected to be qualitatively (but not necessarily quantitatively) similar to each other. Therefore many of our results can be used to understand qualitatively the finite temperature and finite bias behavior of the dot in the absence of the Zeeman field $B$ by replacing $B$ with $V$ or $T$.

\section{LINEAR CONDUCTANCE}

As discussed above, in the vicinity of the singlet-triplet transition the Hamiltonian of the dot can be truncated to that of a two-level system (Eqs. (2) and (5)). The tunneling, Eq. (4), couples the two levels to the two leads, and the four tunneling amplitudes $t_{\alpha, n}$ form a $2 \times 2$ matrix

$$
\hat{t}=\left(\begin{array}{cc}
t_{L,+1} & t_{R,+1} \\
t_{L,-1} & t_{R,-1}
\end{array}\right) .
$$

Consider first the special case when one of the eigenvalues of the matrix $\hat{t}$ is zero, while another one is finite $e^{12}$. Obviously, in this case the dot effectively interacts only with a single species (a single "channel") of conduction electrons. When the dot is in the triplet state, a single electronic channel can screen only half of its $\operatorname{spin}^{24}$. Accordingly, the system should exhibit a quantum phase transition 25 : the ground state changes its symmetry from a singlet to a doublet as $\Delta$ decreases below a certain critical value $\Delta_{C} \sim E_{S}$. The linear conductance $g(\Delta, 0)$ across the system at $T=0$ and $B=0$ is then strongly $\Delta$-dependent12: $g(\Delta, 0) \propto \theta\left(\Delta_{C}-\Delta\right)$. At $\Delta<\Delta_{C}$ (when the dot is in the triplet state) the conductance is a monotonically decreasing function of $B$. To the contrary, at $\Delta>\Delta_{C}$ the conductance as a function of $B$ first increases, and then drops with the increase of $B$. At a fixed finite $B$ the conductance $g(\Delta, B)$ is expected to be a smooth non-monotonic function of $\Delta$ with a broad asymmetric peak near the transition.

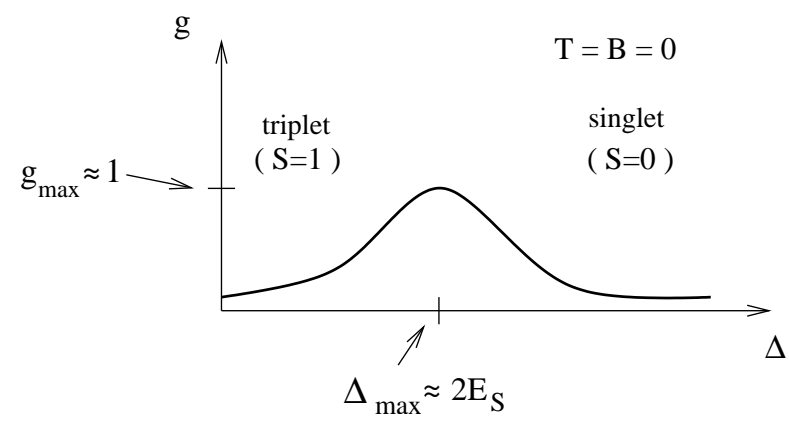

FIG. 3: Sketch of the zero temperature conductance as a function of the splitting between the two dot levels for generic tunneling amplitudes.

In a general situation, however, $\operatorname{det} \hat{t} \neq 0$, and both eigenvalues of the matrix $\hat{t}$ are finite. In this case, the dot is coupled to two electronic channels, which is sufficient in order to completely screen the dot's $\operatorname{spin}^{24}$ even in its triplet state. As a result, the ground state of the system is a singlet at all values of $\Delta^{9.10 .26}$. Accordingly, when the dot is coupled to the leads, the singlet-triplet transition turns to a crossover. As we discuss below, the conductance $g(\Delta, 0)$ at $T=0$ and $B=0$ is then expected to be a smooth non-monotonic function of $\Delta$, slowly (logarithmically) decreasing ${ }^{10}$ at large $\left|\Delta-2 E_{S}\right|$ (see Fig. 3). Far away from the crossover, the conductance is a non-monotonic function of $B$ : it first raises and may reach a value $g \sim 1$, and then drops again as $B$ increases. This has been shown for both the triplet side of the crossover ${ }^{13.27}\left(\Delta \ll E_{S}\right)$, and for the singlet $\left(\Delta \gg E_{S}\right)$ side of it 10.28 . For a fixed small $B$, however, the conductance $g(\Delta, B)$ can be an even more complicated function of $\Delta$ with a local minimum at some $\Delta<E_{S}$ and maxima at the triplet side of the transition $\left(\Delta \ll E_{S}\right)$ and around $\Delta \sim E_{S}$.

In this paper, we focus on a particular limit when both eigenvalues of $\hat{t}$ are finite, but still very different: $|\operatorname{det} \hat{t}| \ll(\operatorname{Tr} \hat{t})^{2}$. In addition, we constrain our considerations to a special subset ${ }^{26.27}$ of the matrices $\hat{t}$ that can be parametrized as

$$
\hat{t}=\frac{1}{\sqrt{2}}\left(\begin{array}{cc}
v_{+1} & v_{+1} \\
v_{-1} & -v_{-1}
\end{array}\right), \quad v_{ \pm 1} \neq 0 .
$$

This tunneling matrix arises, e.g., for a fully symmetric arrangement when the states $n= \pm 1$ are symmetric/antisymmetric under reflection. The choice ([6), although by no means general, nevertheless captures the 
essential physics of the system. At the same time, as we demonstrate shortly below, the choice (6) allows one to express the conductance $g(\Delta, B)$ at $T=0$ in terms of simple phase shifts that can be extracted from the finite size NRG spectra ${ }^{17}$, or in some cases can be related to thermodynamic quantities 26,27 .

Since the ground state of the system is not degenerate, electrons scatter elastically at $T=0$. The scattering amplitudes $S_{s ; \alpha, \alpha^{\prime}}$ of an electron with spin $s$ from lead $\alpha^{\prime}$ to lead $\alpha$ form the scattering matrix $S_{s \alpha ; s^{\prime} \alpha^{\prime}}=\delta_{s s^{\prime}} S_{s ; \alpha, \alpha^{\prime}}$. The $4 \times 4$ unitary matrix $\hat{S}$ can be diagonalized by a rotation in the $R-L$ space to the new basis of channels $n=+1$ and $n=-1$,

$$
U \hat{S} U^{\dagger}=\operatorname{diag}\left\{e^{2 i \delta_{n s}}\right\}, \quad U=e^{i \vartheta \tau^{y}} e^{i \varphi \tau^{z}} .
$$

Here $\tau^{i}$ are the Pauli matrices acting in the $R-L$ space. The linear conductance at $T=0$ is related to the offdiagonal elements of $\hat{S}$ by the Landauer formula,

$$
g=\frac{1}{2} \sum_{s}\left|S_{s ; R L}^{2}\right|
$$

and, by making use of Eqs. (7), can now be expressed via the scattering phase shifts $\delta_{n s}$ at the Fermi energy. In general, the angle $\vartheta$ and thus $g$ depend explicitly on the parameters of the microscopic Hamiltonian. However, with the choice Eq. (6) the tunneling part of the Hamiltonian can be trivially diagonalized with the introduction of even and odd states (corresponding to $\vartheta=\pi / 4, \varphi=0$ )

$$
\left(\begin{array}{c}
c_{+1, k s} \\
c_{-1, k s}
\end{array}\right)=\frac{1}{\sqrt{2}}\left(\begin{array}{c}
a_{R k s}+a_{L k s} \\
a_{R k s}-a_{L k s}
\end{array}\right)
$$

and the Hamiltonian takes the form

$$
H=\sum_{n k s}\left[\xi_{k} c_{n k s}^{\dagger} c_{n k s}+v_{n}\left(c_{n k s}^{\dagger} d_{n s}+\text { H.c. }\right)\right]+H_{\mathrm{dot}} .
$$

The Hamiltonian (8) with $H_{\text {dot }}$ given by Eqs. (2), (5) obviously conserves the total number of fermions in each channel, $\mathcal{N}_{n}=\sum_{k s}\left[c_{n k s}^{\dagger} c_{n k s}+d_{n s}^{\dagger} d_{n s}\right]$, implying that the scattering matrix is diagonal in $n$.

In this case the conductance simply reads ${ }^{13.29}$ :

$$
g=\frac{1}{2} \sum_{s} \sin ^{2}\left(\delta_{+1, s}-\delta_{-1, s}\right) .
$$

Following Nozières, at small $B$ the phase shifts can be expanded to linear order ${ }^{30.31}$. As explained in [30], a simple analysis shows that in the presence of a Zeeman field the conductance at low fields behaves as

$$
g(\Delta, B) \approx g(\Delta, 0)+[1-2 g(\Delta, 0)]\left(\frac{B}{B^{*}(\Delta)}\right)^{2}
$$

where the scale $B^{*}$ is a $\Delta$-dependent energy scale and

$$
g(\Delta, 0)=\sin ^{2}\left[\delta_{+1}(\Delta)-\delta_{-1}(\Delta)\right]
$$

In other words, far away from the transition the conductance should increase with $B$ for $g(\Delta, 0)<g^{*} \approx 1 / 2$ while it should typically decrease in the transition region, where $g(\Delta, 0)>g^{*}$. We have to emphasize that the value $g^{*} \approx 1 / 2$, see Eq. (10), is specific to the choice (6), and not universal.

In the following, we shall compute the phase shifts using the powerful numerical renormalization group and determine the conductance using Eq. (9).

\section{NUMERICAL RG, FINITE SIZE SPECTRA, AND PHASE SHIFTS}

The Numerical Renormalization Group (NRG) was originally developed by Wilson to solve the Kondo problem $^{32}$. In contrast to scaling approaches, it is nonperturbative and does not encounter any logarithmic singularities at low energy. Since then it has been extended to the calculation of dynamical quantities 33.34 and has been applied to a variety of quantum-impurity problems, including transport calculations for interacting quantum $\operatorname{dots} 17,35$.

In principle, the NRG method enables us to compute the expectation value of any local operator. Thus one plausible method to obtain the phase shifts would be to compute the occupation numbers $N_{n s}=\left\langle d_{n s}^{\dagger} d_{n s}\right\rangle$ in the ground state by NRG and then use the Friedel sum rule to get the phase shifts as 3,36 .

$$
\delta_{n s}=\pi N_{n s}
$$

Unfortunately, this procedure turns out to deliver unphysical results, especially on the triplet side. The origin of this problem is probably related to the relatively small bandwidth in our calculations and the large logarithmic corrections that appear on the triplet side: As is obvious from the derivation of the Friedel sum rule ${ }^{3}$, there is, in general, a correction term to Eq. (11) which vanishes in the infinite band width limit. In our calculations, the Kondo temperature is comparable to the bandwidth $D$. Furthermore, on the triplet side of the transition the convergence to the ground state is rather slow due to the large ferromagnetic residual exchange coupling, generic to underscreened Kondo models, that can even lead to singular non-Fermi liquid properties 37 . Therefore we cannot use the Friedel sum rule to determine the conductivity of the dot. Nevertheless, the Friedel sum rule should definitely work in the limit of very large cut-offs. It therefore gives us a useful tool to understand qualitatively the evolution of the phase shifts and the conductance.

Fortunately, as discussed below, we can determine the phase shifts directly from the NRG spectra without making use of the Friedel sum rule ${ }^{16}$. This method has been first used to compute the conductance of a mesoscopic double dot system 17 . To do this, we only have to know that the model flows to a universal Fermi liquid fixed point that can be characterized by four phase shifts. 
To explain the method, let us briefly discuss Wilson's NRG procedure. The key idea of Wilson is to map the original problem to a semi-infinite chain. After this transformation our two-channel Hamiltonian maps onto a system of two parallel tight-binding chains coupled to the dot

$$
\begin{aligned}
H & =H_{\mathrm{dot}}+\sum_{n, s} \tilde{v}_{n}\left(d_{n s}^{\dagger} c_{0, n s}+\text { h.c. }\right) \\
& +\sum_{n, s} \sum_{i=0}^{\infty} \xi_{i}\left(c_{i, n s}^{\dagger} c_{i+1, n s}+\text { h.c. }\right)
\end{aligned}
$$

where the hopping matrix elements decay exponentially, $\xi_{i} \approx \frac{1+\Lambda^{-1}}{2} \Lambda^{-i / 2}$, with $\Lambda$ the NRG discretization parameter. In our calculation we take $\Lambda=3$. The $c_{i, n s}$ represent conduction electron excitations at a length scale $\sim \Lambda^{i / 2}$. The NRG assumes a flat dispersionless density of states for the electrons and all energies are measured in units of the bandwidth $D$. The operator $c_{0, n s}$ is simply the local field operator defined as $c_{0, n s} \equiv \frac{1}{\sqrt{2}} \int_{k} d k c_{k n s}$, and it is only this operator that tunnel-couples to the interacting impurity part, $H_{\text {dot }}$. Note also that the discretization procedure somewhat renormalizes the original parameters of the Hamiltonian, which are therefore not identical to the ones in Eq. (6).

Eq. 12 can be rewritten in a more inspiring way as

$$
\begin{aligned}
\tilde{H}_{N+1} & =\Lambda^{1 / 2} \tilde{H}_{N}+\sum_{n, s}\left(c_{N n s}^{\dagger} c_{N+1 n s}+\text { h.c. }\right) \\
H & =\lim _{N \rightarrow \infty} \frac{1+\Lambda^{-1}}{2} \Lambda^{-(N-1) / 2} \tilde{H}_{N}
\end{aligned}
$$

where we have introduced the rescaled Hamiltonians

$$
\begin{aligned}
\tilde{H}_{N} & \equiv \frac{2}{1+\Lambda^{-1}} \Lambda^{(N-1) / 2} H_{N}, \\
H_{N} & \equiv H_{\mathrm{dot}}+\sum_{n, s} \tilde{v}_{n}\left(d_{n s}^{\dagger} c_{0, n s}+\text { h.c. }\right) \\
& +\sum_{n, s} \sum_{i=0}^{N-1} \xi_{i}\left(c_{i, n s}^{\dagger} c_{i+1, n s}+\text { h.c. }\right),
\end{aligned}
$$

and $H_{0}$ denotes the impurity Hamiltonian, $H_{\text {dot }}$, tunnelcoupled to $c_{0, p s}$. Eq. (14) can be solved by iterative diagonalization, by keeping in each step only the lowest, most relevant levels. In our calculation, conservation of total charge and the $z$-component of the total spin

$$
\begin{aligned}
Q & =\sum_{n, s} \sum_{i=0}^{\infty}\left(c_{i, n s}^{\dagger} c_{i, n s}-1 / 2\right) \\
S_{z} & =\sum_{n, s} \sum_{i=0}^{\infty} s c_{i, n s}^{\dagger} c_{i, n s}
\end{aligned}
$$

have been exploited to increase numerical efficiency.

A typical example of the spectrum of $\tilde{H}_{N}$ vs. iteration number is shown in Fig. 4 At large iteration numbers (i.e. small energy scales), the spectrum remains invariant, indicating the presence of a stable field theory (fixed point) which describes the low energy behavior of our model.

To interpret the spectrum, we have to keep in mind that in the NRG procedure the length scale $L$ of the system corresponds to $L \sim \Lambda^{N / 2}$, and therefore, apart from some multiplicative factor, the spectrum of $\tilde{H}_{N}$ is the spectrum of a system of size $L$ in units of $2 \pi v_{F} / L$. This fixed point NRG spectrum is universal and can be characterized by boundary conformal field theories 38 . In our case the fixed point spectrum is that of a Fermi liquid, and is therefore identical to that of the following simple model

$$
H_{\mathrm{fp}}=\sum_{n, s} \int_{-L / 2}^{L / 2} d x \psi_{n s}^{\dagger}(x)\left(-i \partial_{x}+U_{n s} \delta(x)\right) \psi_{n s}(x)
$$

where the $\psi_{n s}(x)$ 's denote chiral fermionic fields obeying periodic and anti-periodic boundary conditions for even and odd $N$, respectively. This statement means that the full many body-spectrum of our model is identical to that of the following simple effective Hamiltonian (in Fourier space):

$$
H_{\mathrm{fp}}=\frac{2 \pi}{L} \sum_{n, s} \sum_{q}\left(q-\frac{\delta_{n, s}}{\pi}\right) \psi_{q, n s}^{\dagger} \psi_{q, n s},
$$

where the phase shifts $\delta_{n, s}$ are related to the couplings $U_{n s}$ and $q$ takes integer (half-integer) values for even (odd) iterations. In more precise terms:

$$
\lim _{N \rightarrow \infty} \tilde{H}_{N}=C \frac{L}{2 \pi} H_{\mathrm{fp}}
$$

where $C$ is a $\Lambda$-dependent constant to be determined numerically. The quantum numbers $Q$ and $S_{z}$ in the



FIG. 4: A typical NRG level flow on the triplet side for $E_{C}=1, E_{S}=0.25, \Delta=0.2, v_{+1}=0.67, v_{-1}=0.37$ and $B=0.01$. The results were obtained with $\Lambda=3$ and keeping $N=1000$ levels. 


\begin{tabular}{c|cccc}
\hline \hline state & $\tilde{E}_{\mathrm{NRG}} / C$ & $L E_{\mathrm{fp}} / 2 \pi$ & $Q$ & $S_{z}$ \\
\hline 1 & 0.1288 & $1-\frac{\delta_{\uparrow}}{\pi}=0.1288$ & 1 & $1 / 2$ \\
2 & 0.1757 & $\frac{\delta_{\downarrow}}{\pi}=0.1757$ & -1 & $1 / 2$ \\
3 & 0.3045 & $1-\frac{\delta_{\uparrow}}{\pi}+\frac{\delta_{\downarrow}}{\pi}=0.3045$ & 0 & 1 \\
4 & 0.3661 & $\frac{\tilde{\delta}_{\downarrow}}{\pi}=0.3661$ & -1 & $1 / 2$ \\
5 & 0.4157 & $1-\frac{\delta_{\uparrow}}{\pi}=0.4157$ & 1 & $1 / 2$ \\
6 & 0.4949 & $1+\frac{\tilde{\delta}_{\downarrow}}{\pi}-\frac{\delta_{\uparrow}}{\pi}=0.4949$ & 0 & 1 \\
7 & 0.5418 & $\frac{\tilde{\delta}_{\downarrow}}{\pi}+\frac{\delta_{\downarrow}}{\pi}=0.5418$ & -2 & 1 \\
8 & 0.5445 & $2-\frac{\tilde{\delta}_{\uparrow}}{\pi}-\frac{\delta_{\uparrow}}{\pi}=0.5445$ & 2 & 1 \\
9 & 0.5843 & $\frac{\delta_{\uparrow}}{\pi}=0.5843$ & -1 & $-1 / 2$ \\
10 & 0.5914 & $1-\frac{\tilde{\delta}_{\uparrow}}{\pi}+\frac{\delta_{\downarrow}}{\pi}=0.5914$ & 0 & 1 \\
\hline \hline
\end{tabular}

TABLE I: Comparison of the even iteration fixed point NRG spectrum in Fig. 4 and that of the effective field theory, Eq.20] We used $C=1.6023$, and the phase shifts were $\frac{\delta_{\downarrow}}{\pi}=0.1757$, $\frac{\tilde{\delta}_{\downarrow}}{\pi}=0.3661, \frac{\tilde{\delta}_{\uparrow}}{\pi}=0.5843$, and $\frac{\delta_{\uparrow}}{\pi}=0.8712 . H_{\mathrm{fp}}$ reproduces all the quantum numbers correctly and the NRG spectrum with a four digit precision.

fixed point theory are defined as

$$
\begin{aligned}
Q^{\mathrm{fp}} & =\sum_{q, n, s}: \psi_{q, n s}^{\dagger} \psi_{q, n s}:, \\
S_{z}^{\mathrm{fp}} & =\sum_{q, n, s} s \psi_{i, n s}^{\dagger} \psi_{i, n s},
\end{aligned}
$$

where $: \ldots$ : denotes normal ordering with respect to the ground state.

To illustrate how well this procedure works we have enumerated in Table \ the lowest eigenstates and their quantum numbers in the NRG spectrum together with those of the effective Hamiltonian $H_{\mathrm{fp}}$ : All quantum numbers are in agreement and the eigenvalues match up to four digits. In our calculations we have not kept track of the conserved quantum numbers $N_{n}$. Therefore, while we could tell the values of the two spin up and spin down phase shifts, $\delta_{s}$ and $\tilde{\delta}_{s}$, in some cases we were unable to tell to which channel they belonged. Since, however, Eq. (9) is symmetrical under the exchange of $n= \pm$, this caused no problem in the calculation of the conductance.

This method enables us to extract the phase shifts $d i$ rectly from the finite size spectrum. The great advantage of this procedure is that it works directly on the fixed point Hamiltonian and therefore eliminates the problem of high energy degrees of freedom that occurred when using the Friedel sum rule. This method can be applied to any problem where the ground state is that of a Fermi liquid.

\section{RESULTS}

In Fig. 5 we have plotted the typical splitting dependence of the phase shifts for $B=0$ and the corresponding conductance. The qualitative behavior of the phase shifts can be understood in terms of the Friedel sum rule Eq. (11) as follows: On the triplet side of the transition the spin of the dot is completely screened by the electrons in the leads, which corresponds to a phase shift $\pi / 2$ in both channels. On the singlet side, however, both dot electrons occupy the level $n=-1$. Therefore the phase shift in channel $n=-1$ should approach $\delta_{-1, s} \approx \pi$ on this side, while the phase shift $\delta_{+1, s}$ must go to zero. By Eq. (9) this implies that the conductance must approach 0 on both sides of the transition while it has a maximum $g_{\max } \sim 1$ around $\delta_{-1, s}-\delta_{1, s} \approx \pi / 2$. Note that the transition point is shifted with respect to the bare value $\Delta=2 E_{S}=0.5$ for the isolated dot due to correlation effects.

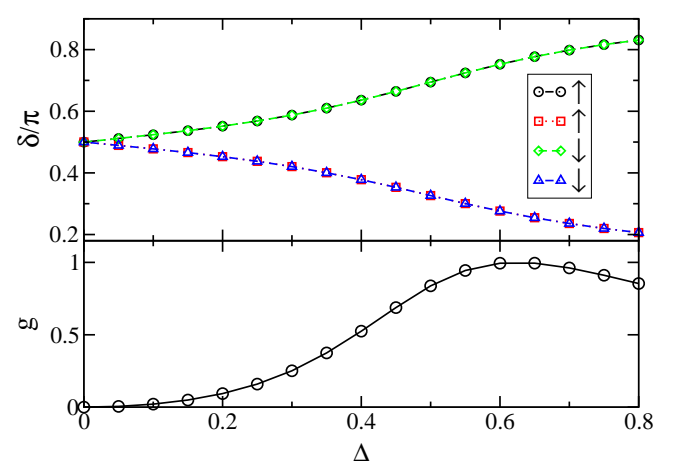

FIG. 5: Phase shifts (top) and corresponding conductance (bottom) as a function of orbital splitting $\Delta$ in the absence of the Zeeman field at temperature $T=0$. Parameters are chosen as $E_{C}=1, E_{S}=0.25, v_{+1}=0.67$ and $v_{-1}=0.50$.

Application of a Zeeman field $B$ has a more complex effect, which to some degree has been discussed in [13]. On the triplet side, $\Delta \ll E_{s}$ we have two exponentially separated Kondo scales, $T_{K}^{>} \gg T_{K}^{<}$. For Zeeman fields $B \ll T_{K}^{>}, T_{K}^{<}$all four phase shifts approximately equal $\pi / 2$, corresponding to a reduced conductance. However, for intermediate fields $T_{K}^{<} \ll B \ll T_{K}^{>}$, the Kondo effect in channel $n=-1$ is suppressed, and therefore the phase shifts in this channel are expected to be around $\delta_{n=-1, \uparrow} \approx \pi$ and $\delta_{n=-1, \downarrow} \approx 0$, while $\delta_{n=+1, \uparrow} \approx \delta_{n=+, \downarrow} \approx$ $\pi / 2$, leading to a conductance $g \sim 1$. Finally, for even larger fields, $B \gg T_{K}^{<}, T_{K}^{>}$, both Kondo effects are suppressed, the phase shifts become $\delta_{n=-1, \downarrow} \approx \delta_{n=1, \downarrow} \approx 0$ and $\delta_{n=-1, \uparrow} \approx \delta_{n=1, \uparrow} \approx \pi$, and the conductance decreases again to 0 . This behavior is shown in Fig. [6

As we already mentioned in Section III in the experiment [1] the Zeeman field was non-negligible. It is therefore instructive to study the splitting-dependence of the conductance in the presence of a finite Zeeman field $B$. This is shown in Fig. (7) In a finite magnetic field $g(\Delta, B=$ fixed $)$ can show a very surprising $\Delta$ dependence for some values of $B$, and may even have a dip on the triplet side. As we explain below, this dip appears because $T_{K}^{<}$is very sensitive to the distance from the transition point, and therefore we may cross over from the regime $T_{K}^{>}, T_{K}^{<}>B$ to $T_{K}^{>}>B>T_{K}^{<}$as we 


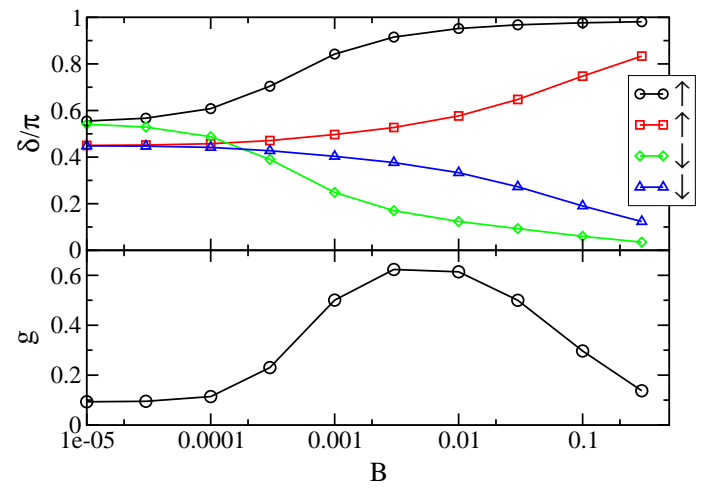

FIG. 6: Phase shifts (top) and corresponding conductance (bottom) as a function of Zeeman field $B$ on the triplet side of the transition at zero temperature. Parameters are $E_{C}=1$, $E_{S}=0.25, \Delta=0.4, v_{+1}=0.67$ and $v_{-1}=0.25$.

are getting farther away from the transition region. For intermediate Zeeman fields $B$ the conductance has the shape of a smeared step function that becomes sharper as the ratio $v_{+} / v_{-}$increases and resembles the one found in [12].

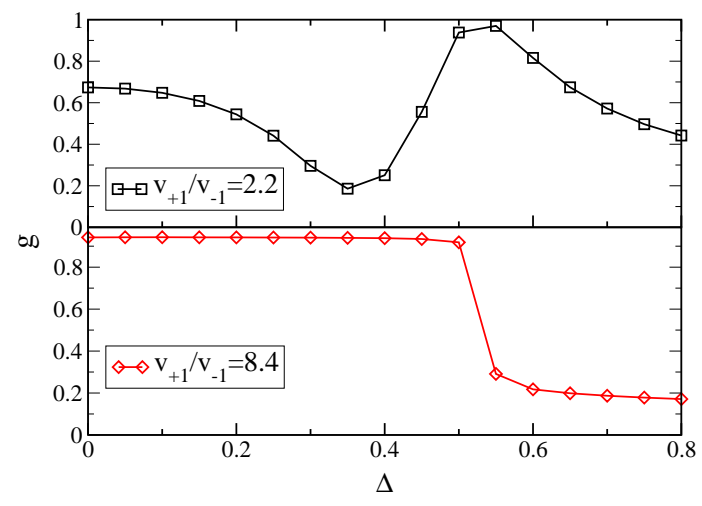

FIG. 7: Conductance as a function of $\Delta$ for a fixed value $B=$ $10^{-3}$ of the Zeeman field and coupling asymmetry $v_{+1} / v_{-1}=$ 2.2 (top) and $v_{+1} / v_{-1}=8.4$ (bottom). The other parameters are $E_{C}=1, E_{S}=0.25, v_{+1}=0.67$.

The effect of a Zeeman field on the conductance should qualitatively resemble that of a finite source-drain bias or temperature. We can thus use the $B$-dependence of the conductance for fixed fixed values of $\Delta$ to identify the second energy scale that appears as the width of a dip in the zero-bias anomaly in the experiment 11. The $B$-dependent conductance for three different values of $\Delta$ is shown in Fig. 8 The conductance first increases for small Zeeman fields $B$ on both sides of the transition, but it is suppressed for larger values of $B$. However, it decreases monotonically in the transition region, where $g>g^{*}$ (see Eq. 10).

We can then identify the low-energy scale $B^{*}$ by fixing $\Delta$ and measuring the curvature of $g(B, \Delta)$. This is

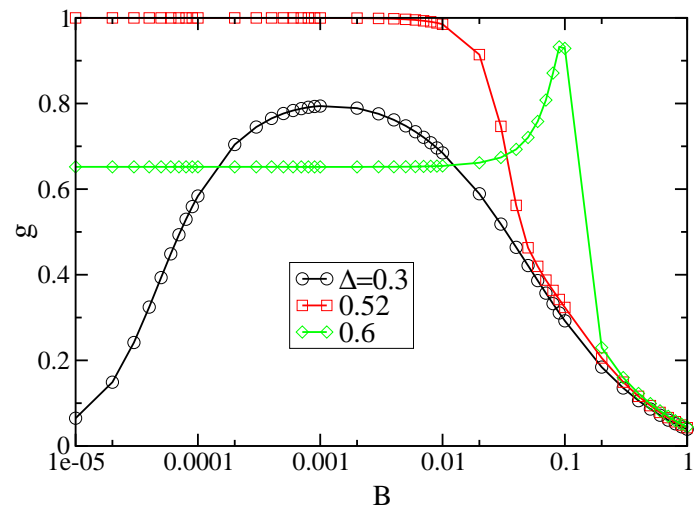

FIG. 8: Linear conductance versus in-plane magnetic field $B$ for different values of the orbital splitting and the same parameters as in Fig. 6] Notice the non-monotonic behavior ("dip") on both the triplet $(\Delta=0.3)$ and singlet $(\Delta=0.6)$ sides of the cross-over. In the middle of the transition $(\Delta=0.52)$ the conductance is $g=1$ at $B=0$, and decreases monotonically as a function of magnetic field.

shown in Fig. 9 On the singlet side of the transition $B^{*}$ increases approximately linearly with $\Delta$, and practically corresponds to the energy splitting of the singlet and triplet multiplets. Intuitively this can be understood in the following way: Applying a Zeeman field one gradually pulls down the $S_{z}=-1$ state of the excited triplet state. This becomes degenerate with the singlet state if $B$ is approximately equal to the splitting between the triplet and the singlet states of the dot. At this special value of $B$ a Kondo effect can occur between the ground state singlet and the $S_{z}=-1$ triplet state ${ }^{28}$, giving a conductance maximum as a function of $B$. Indeed, we can see in Fig. 8 that on the singlet side $(\Delta=0.6)$ the maximum conductance is indeed approximately $g \approx 1$, indicative of the Kondo resonance. Therefore, on the singlet side $B^{*}$ must be proportional to the renormalized (by quantum fluctuations) singlet-triplet splitting.

The origin of the dip on the triplet side is very different, as explained above. Far away from the transition, the spin $S$ of the dot is screened by two consecutive Kondo effects. An intermediate magnetic field suppresses the Kondo effect with the smaller Kondo temperature, $T_{K}^{<}$, and leads to a large, almost unit conductance. Therefore, in this region, the energy scale $B^{*}$ should practically correspond to the smallest of these two Kondo temperatures, i.e. the one with smaller hybridization $v_{<}$. Far away from the triplet side, $T_{K}^{<}$does not depend strongly on the presence of the singlet. However, in the vicinity of the singlet-triplet transition, quantum fluctuations to the singlet state are extremely important: They mix all four dot states, renormalize the value of $v_{<}$, and generate a strongly correlated state with a Kondo scale that is determined by the hybridization to the more strongly coupled channel, $v_{>}$. As a consequence, in the vicinity of the transition these fluctuations strongly renormalize the value of $v_{<}$and thus influence $B^{*} \sim T_{K}^{<}$. This is shown 


\section{in Fig. 9}

For the parameters of the figure the second Kondo scale would be very small in the absence of fluctuations to the singlet state. Therefore, $B^{*}$ is extremely sensitive to the distance from the triplet-singlet transition, which sets the renormalized value of $v_{<}$, and thus that of $T_{K}^{<} \sim B^{*}$. Far from the transition the effect of the fluctuations to the singlet state is small, and they do not renormalize $T_{K}^{<} \sim B^{*}$ substantially. Thus $T_{K}^{<}$saturates on the triplet side and shows only a weak $\Delta$-dependence there, in complete agreement with the experimental results of Ref. 11]. However, for larger ratios of $v_{+1} / v_{-1}$, the saturation value $T_{K}^{<}\left(\Delta_{\mathrm{ST}} \ll 0\right)$ becomes so small that it can hardly be observed.

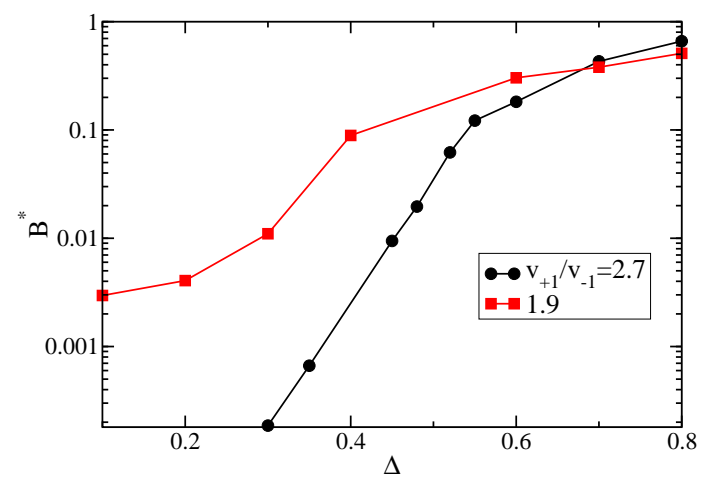

FIG. 9: Low-energy scale $B^{*}$ for $E_{C}=1, E_{S}=0.25$ and $v_{+1}=0.67$, as determined from the curvature of $g(\Delta, B)$ for small Zeeman field $B$. Note the saturation of $B^{*}$ on the triplet side for the larger $v_{-1}$.

In Fig. 10 we show the $\Delta$-dependence of the conductance for three different values of $B$. The conductance has a similar behavior for the two lower values of $B$. In these cases, far away on the triplet side, we have a situation where $T_{K}^{<}<B<T_{K}^{>}$. The smaller Kondo scale, $T_{K}^{<}$, gradually increases as we approach the transition point, and therefore the conductance decreases $T_{K}^{<}$becomes larger than $B$, thus giving a dip on the triplet side of the transition.

The third case is very different. Here $B=0.1$ is larger than both Kondo scales, and therefore the bump on the triplet side is suppressed. Even the maximum associated with the triplet-singlet transition is shifted to larger values of $\Delta$. We suspect that in this case the bump has an entirely different origin and is due to the Kondo effect associated with the degeneracy of the $S_{z}=-1$ triplet state and the singlet state ${ }^{28}$.

\section{CONCLUSIONS}

We have studied transport through a lateral quantum dot in the vicinity of a singlet-triplet transition in the ground state. Our calculation is based on the numerical renormalization group, which enables us to calculate the full many-body spectrum of the dot. To determine

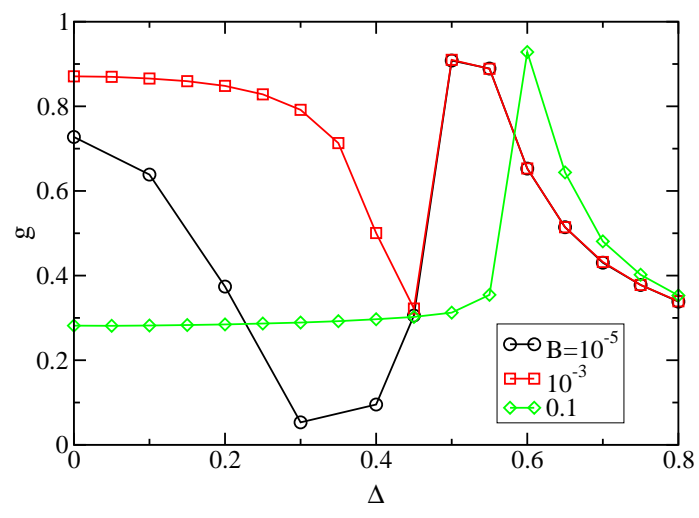

FIG. 10: Linear conductance versus level splitting for different in-plane magnetic fields $B$ and a fixed ratio $\tilde{v}_{+1} / \tilde{v}_{-1}=$ 2.6. The other parameters are the same as in Fig. [6]

the conductance we used a new method, first outlined in [17], where we extract the conduction electron phase shifts with high accuracy directly from the NRG spectrum and use them in combination with the Landauer formula to determine the conductance at zero temperature. The only requirement for this technique to work is that the system considered must have a Fermi liquid ground state with the phase shifts being well-defined quantities.

In our calculations have modeled the quantum dot by a two-level Anderson impurity Hamiltonian with asymmetric couplings to the left and right single-mode lead. Our results show that at $T=0$ the sharp quantum phase transition that occurs for symmetric couplings becomes a crossover at finite asymmetry. As a function of level splitting, the conductance shows a characteristic maximum ("bump") at the crossover point and decreases on both the singlet and the triplet side.

In order to model the effect of finite temperature or bias voltage, we have applied an in-plane magnetic field $B$ leading to a Zeeman-split triplet. We find that on both the singlet and the triplet side, the conductance has a non-monotonic behavior as a function of the Zeeman field, thus leading to a characteristic "dip" at low $B$. Because of the qualitative equivalence between $B$ and finite bias voltage, we expect this structure to appear in the differential conductance $d I / d V$ as well. The associated low-energy scale (dip width) increases linearly on the singlet side, where it corresponds to the renormalized singlet-triplet splitting. On the triplet side, it decreases rapidly with increasing distance from the degeneracy point, which is in agreement with two-stage Kondo screening of the triplet.

These findings are consistent with the experimental results of van der Wiel et al. [11], where a dip in $d I / d V$ was indeed found on both sides of the singlet-triplet transition. On the other hand, in the measurements of Kogan et al. 14], the dip structure was only observed on the singlet side, indicating a weak asymmetry below experimental resolution.

We expect our results to be relevant also for trans- 
port through other multi-level Kondo systems like carbon nanotubes or molecules.

Acknowledgements: We are grateful to M. Pustilnik, L. Glazman, M. Kastner, G. Granger and A. Kogan for illuminating discussions. This research has been supported by NSF Grants DMR-99-81283, DMR-02-33773, Hungarian Grants OTKA F030041, T038162, and the EU 'Spintronics' Research Training Network. W.H. acknowledges financial support from the German Science Foundation (DFG). G.Z. is a Bolyai fellow.
* Electronic address: hofstett@cmt.harvard.edu

† Electronic address: zarand@newton.phy.bme.hu

1 D. Goldhaber-Gordon, H. Shtrikman, D. Mahalu, D. Abusch-Magder, U. Meirav, M.A. Kastner, Nature (London) 391, 156 (1998); S.M. Cronenwett, T.H. Oosterkamp, and L.P. Kouwenhoven, Science 281, 540 (1998); J. Schmid, J. Weis, K. Eberl, K.von Klitzing, Physica B 256-258, 182 (1998).

2 L.I. Glazman and M.E. Raikh, JETP Lett. 47, 452 (1988); T.K. Ng, P.A. Lee, Phys. Rev. Lett. 61, 1768 (1988).

3 A.C. Hewson, The Kondo Problem to Heavy Fermions, Cambridge University Press, Cambridge (1993).

4 L.P. Kouwenhoven, C.M. Marcus, P.L. McEuen, S. Tarucha, R.M. Westervelt, and N.S. Wingreen, in Mesoscopic Electron Transport, eds. L.L. Sohn, L.P. Kouwenhoven, and G. Schön, NATO ASI Series E - vol. 345 (Kluwer, Dordrecht, 1997), pp. 105-214.

5 Y. Ji, M. Heiblum, D. Sprinzak, D. Mahalu, and H. Shtrikman, Science 290, 779 (2000).

${ }^{6}$ S. Tarucha, D.G. Austing, Y. Tokura, W.G. van der Wiel, and L.P. Kouwenhoven, Phys. Rev. Lett. 84, 2485 (2000).

7 S. Sasaki, S. De Franceschi, J. M. Elzerman, W. G. van der Wiel, M. Eto, S. Tarucha, and L. P. Kouwenhoven, Nature 405, 764 (2000).

8 M. Eto and Yu.V. Nazarov, Phys. Rev. Lett. 85, 1306 (2000); Phys. Rev. B 66, 153319 (2002).

9 M. Pustilnik and L.I. Glazman, Phys. Rev. Lett. 85, 2993 (2000).

10 M. Pustilnik and L.I. Glazman, Phys. Rev. B 64, 045328 (2001).

11 W.G. van der Wiel, S. De Franceschi, J.M. Elzerman, S. Tarucha, L.P. Kouwenhoven, J. Motohisa, F. Nakajima, and T. Fukui, Phys. Rev. Lett. 88, 126803 (2002).

12 W. Hofstetter and H. Schoeller, Phys. Rev. Lett. 88, 016803 (2002).

13 M. Pustilnik and L.I. Glazman, Phys. Rev. Lett. 87, 216601 (2001).

14 A. Kogan, G. Granger, M.A. Kastner, D. GoldhaberGordon, Hadas Shtrikman, D. Mahalu, and U. Meirav, Phys. Rev. B 67, 113309 (2003).

15 G. Zaránd, A. Brataas, and D. Goldhaber-Gordon, Solid State Comm. 126, 463 (2003).

16 I. Affleck, A. Ludwig, H. Pang, and D.L. Cox, Phys. Rev. B 45, 7918 (1992).
17 L. Borda, G. Zarand, W. Hofstetter, B.I. Halperin, and J. von Delft, Phys. Rev. Lett. 90, 026602 (2003).

18 K. A. Matveev Phys. Rev. B 51, 1743 (1995); K. Flensberg, Phys. Rev. B 48, 11156 (1993).

19 L.I. Glazman, J. Low Temp. Phys. 118, 247 (2000).

20 I.L. Aleiner, P.W. Brouwer, and L.I. Glazman, Physics Reports 358, 309 (2002).

21 Strictly speaking, Eq. (2) is fully justified only for very large dots with chaotic single-particle states ${ }^{20}$. For ballistic motion of electrons within the dot the magnitude of the terms not included in Eq. (2) is of the order of $N^{-1 / 4} \delta E$. Inclusion of these terms is not expected to change the results qualitatively ${ }^{10}$.

22 G. Falci, G. Schön, G.T Zimanyi, Phys. Rev. Lett. 74, 3257 (1995).

23 M. Pustilnik, L.I. Glazman, D.H. Cobden, and L.P. Kouwenhoven, Lecture Notes in Physics, 579, 3 (2001).

24 P. Nozières and A. Blandin, J. Phys. (Paris) 41, 193 (1980).

25 M. Vojta, R. Bulla, and W. Hofstetter, Phys. Rev. B 65, 140405 (2002).

${ }^{26}$ W. Izumida, O. Sakai, and S. Tarucha, Phys. Rev. Lett. 87, 216803 (2001).

27 W. Izumida, O. Sakai, and Y. Shimizu, J. Phys. Soc. Jpn. 67, 2444 (1998).

${ }^{28}$ M. Pustilnik, Y. Avishai, and K. Kikoin, Phys. Rev. Lett. 84, 1756 (2000)

29 A. Georges and Y. Meir, Phys. Rev. Lett. 82, 3508 (1999).

30 M. Pustilnik, L.I. Glazman, and W. Hofstetter, cond-mat/0304688

31 P. Nozières, J. Low Temp. Phys. 17, 31 (1974).

32 K.G. Wilson, Rev. Mod. Phys. 47, 773 (1975).

33 T.A. Costi, A.C. Hewson, and V. Zlatić, J. Phys.: Cond. Mat. 6, 2519 (1994).

${ }^{34}$ W. Hofstetter, Phys. Rev. Lett. 85, 1508 (2000).

35 W. Hofstetter, Adv. in Sol. State Phys. 41: pp. 27-38 (2001).

36 D.C. Langreth, Phys. Rev. 150, 516 (1966).

37 P. Coleman and C. Pepin, cond-mat/0302001

38 For a review see e.g. A.W.W. Ludwig, in Low-dimensional quantum field theories for condensed matter physicists, edited by S. Lundqvist, G. Morandi, and Yu Lu, World Scientific, Singapore, (1995). 